Publ. Mat. 63 (2019), 219-240

DOI: 10.5565 /PUBLMAT6311907

\title{
GROWTH ALTERNATIVE FOR HECKE-KISELMAN MONOIDS
}

\author{
Arkadiusz MȨCEL AND JAN OKNińSKI
}

\begin{abstract}
The Gelfand-Kirillov dimension of Hecke-Kiselman algebras defined by oriented graphs is studied. It is shown that the dimension is infinite if and only if the underlying graph contains two cycles connected by an (oriented) path. Moreover, in this case, the Hecke-Kiselman monoid contains a free noncommutative submonoid. The dimension is finite if and only if the monoid algebra satisfies a polynomial identity.
\end{abstract}

2010 Mathematics Subject Classification: 16P90, 16S15, 16S36, 16S99, $20 \mathrm{M} 05$.

Key words: Oriented simple graph, Hecke-Kiselman monoid, Gelfand-Kirillov dimension, growth alternative.

\section{Introduction}

Let $\Theta$ be an arbitrary finite simple digraph with $n$ vertices $\{1, \ldots, n\}$. So, in other words, it is a simple graph in which two vertices can be connected by an oriented edge (an arrow) or an unoriented edge (an edge). In [10] the following monoid $\mathrm{HK}_{\Theta}$ associated with $\Theta$ has been defined, by specifying generators and the set of defining relations:

(i) $\mathrm{HK}_{\Theta}$ is generated by idempotents $e_{i}^{2}=e_{i}$, where $1 \leq i \leq n$;

(ii) if the vertices $i, j$ are not connected in $\Theta$, then $e_{i} e_{j}=e_{j} e_{i}$;

(iii) if $i, j$ are connected by an arrow $i \rightarrow j$ in $\Theta$, then $e_{i} e_{j} e_{i}=e_{j} e_{i} e_{j}=$ $e_{i} e_{j}$

(iv) if $i, j$ are connected by an edge in $\Theta$, then $e_{i} e_{j} e_{i}=e_{j} e_{i} e_{j}$.

If the graph $\Theta$ is unoriented (has no arrows), the monoid $\mathrm{HK}_{\Theta}$ is isomorphic to the so-called 0 -Hecke monoid $H_{0}(W)$, where $W$ is the Coxeter group of the graph $\Theta$, see [8]. The latter monoid plays an important role in representation theory. In the case $\Theta$ is oriented (all edges are arrows) and acyclic, the monoid $\mathrm{HK}_{\Theta}$ is finite and it is a homomorphic image of the so-called Kiselman monoid $K_{n}$, see [10], [13]. 
The monoid $\mathrm{HK}_{\Theta}$ has been studied in particular in $[\mathbf{2}],[\mathbf{3}],[\mathbf{7}],[\mathbf{9}]$. So far, the emphasis has been concentrated on three problems, which still remain open in full generality: on the description of all graphs $\Theta$, for which the monoid $\mathrm{HK}_{\Theta}$ is finite; on the existence of faithful representations of the monoid $\mathrm{HK}_{\Theta}$ in the multiplicative semigroup $\mathbb{M}_{n}(\mathbb{Z})$ of matrices over the ring of integers; and whether $\mathrm{HK}_{\Theta}$ is always a $\mathcal{J}$-trivial monoid. The latter means that the Green $\mathcal{J}$-relation on $\mathrm{HK}_{\Theta}$ is trivial; in other words: $\mathrm{HK}_{\Theta} s \mathrm{HK}_{\Theta}=\mathrm{HK}_{\Theta} t \mathrm{HK}_{\Theta}$ implies that $s=t$, for $s, t \in \mathrm{HK}_{\Theta}$. The last problem has been solved in the affirmative in the case $\Theta$ is oriented, as well as in the case $\Theta$ is unoriented, and also in certain mixed cases (see $[\mathbf{3}]$ ). This problem is essential for the representation theory of such monoids, since representations of $\mathcal{J}$-trivial monoids admit a very satisfactory approach, see $[\mathbf{8}]$.

The present paper is motivated by a study of the growth of HeckeKiselman monoids, or in other words of the Gelfand-Kirillov dimension of the semigroup algebras $A_{\Theta}:=\mathrm{K}\left[\mathrm{HK}_{\Theta}\right]$ over a field $\mathrm{K}$. The main aim is to establish the growth alternative for $A_{\Theta}$ in the case when $\Theta$ is an oriented graph. In other words, we show that the growth is either polynomial or exponential. Clearly, the Gelfand-Kirillov dimension of $A_{\Theta}$ is 0 if and only if the monoid $\mathrm{HK}_{\Theta}$ is finite. In the case of oriented graphs $\Theta$ this means exactly that the graph is acyclic, see $[\mathbf{1 3}]$.

Our main result reads as follows.

Theorem 1. Assume that $\Theta$ is a finite oriented simple graph. The following conditions are equivalent.

(1) $\Theta$ does not contain two different cycles connected by an oriented path of length $\geq 0$.

(2) $A_{\Theta}$ is a PI-algebra.

(3) $\operatorname{GKdim}\left(A_{\Theta}\right)<\infty$.

(4) The monoid $\mathrm{HK}_{\Theta}$ does not contain a free submonoid of rank 2 .

Here, if $\Theta=(V(\Theta), E(\Theta))$ is an oriented graph with the set of vertices $V(\Theta)$ and the set of arrows $E(\Theta)$, then we say that two induced subgraphs $\Theta_{1}$ and $\Theta_{2}$ of $\Theta$ are:

- connected by a path of length 0 , if $V\left(\Theta_{1}\right) \cap V\left(\Theta_{2}\right) \neq \emptyset$;

- connected by a path of length 1 , if $V\left(\Theta_{1}\right) \cap V\left(\Theta_{2}\right)=\emptyset$ and there exist $w \in V\left(\Theta_{1}\right), v \in V\left(\Theta_{2}\right)$, and an arrow $w \rightarrow v$, or $v \rightarrow w$, in the graph $\Theta$;

- connected by a path of length $k>1$, if the above two cases do not occur and if $k$ is the minimal integer such that there exists 
a sequence of vertices $w_{0}, \ldots, w_{k} \in V(\Theta)$ such that $w_{0} \in V\left(\Theta_{1}\right)$, $w_{k} \in V\left(\Theta_{2}\right), w_{i} \notin V\left(\Theta_{1}\right) \cup V\left(\Theta_{2}\right)$ for $0<i<k$ and in $\Theta$ there exist arrows $w_{i} \rightarrow w_{i+1}$, or there exist arrows $w_{i+1} \rightarrow w_{i}$, for all $i=0,1, \ldots, k-1$.

We note that the growth alternative result for $A_{\Theta}$ in the case when the graph $\Theta$ is unoriented follows from classical results on Coxeter groups and monoids. As mentioned above, in this case $\mathrm{HK}_{\Theta}=H_{0}(W)$, where $W$ is the Coxeter group of the graph $\Theta$. The growth of $W$ and $H_{0}(W)$ is actually the same, which follows from the word property theorem of Tits [19], see also [20, Theorem 1]. More precisely, the reduced words for the Coxeter group and Coxeter monoid are the same, and two reduced words represent the same element of the Coxeter group if and only if they represent the same element of the Coxeter monoid. The theorem by de la Harpe [11] states, however, that the growth of the Coxeter group $W$ is either polynomial, in the case when $W$ is finite or affine (and $W$ is abelian-by-finite in this case), or exponential in other cases. Moreover, it is known that there exists a finite automaton that recognizes the language of normal forms of elements of a Coxeter group [6], hence also the language of normal forms of elements of the corresponding monoid $H_{0}(W)$. In our particular case, by a standard argument (see [22, p. 97]) one obtains the following result.

Theorem 2. Assume that $\Theta$ is a finite unoriented simple graph. The following conditions are equivalent.

(1) $\Theta$ is a disjoint union of Dynkin and extended Dynkin diagrams.

(2) $\operatorname{GKdim}\left(A_{\Theta}\right)<\infty$.

(3) The monoid $\mathrm{HK}_{\Theta}$ does not contain a free submonoid of rank 2.

Clearly, Theorems 1 and 2 imply certain conditions concerning the Gelfand-Kirillov dimension of the algebra $A_{\Theta}$, when $\Theta$ is any mixed digraph. However, we do not dwell on those details in this paper.

A similar growth alternative has been known in several other contexts; in particular, it holds in the class of monomial algebras, that provide a rich area of examples of algebras with a particular growth behavior and have been used to answer several questions on the Gelfand-Kirillov dimension of arbitrary algebras. Recall that for an ideal $I$ of the free monoid $\langle X\rangle$ on a set $X$ one defines $\mathrm{K}_{0}[\langle X\rangle / I]=\mathrm{K}[\langle X\rangle] / \mathrm{K}[I]$. The result obtained independently by Anick [1] and Ufnarovskii [21] can be stated as follows (see also [15, Theorem 24.19]). 
Theorem 3. Assume that $I$ is a finitely generated ideal of the free monoid $\langle X\rangle$ on a finite set $X$. Then the following conditions are equivalent.

(1) $\operatorname{GKdim}\left(\mathrm{K}_{0}[\langle X\rangle / I]\right)<\infty$.

(2) $\langle X\rangle / I$ does not contain a free submonoid of rank 2.

(3) $\mathrm{K}_{0}[\langle X\rangle / I]$ is a PI-algebra.

Moreover, if these conditions are satisfied, then $\operatorname{GKdim}\left(\mathrm{K}_{0}[\langle X\rangle / I]\right)$ is an integer.

In certain more general classes of algebras (for example, algebras admitting a finite Gröbner basis, or in the case of automaton algebras) similar results have been obtained by associating to the algebra, or to the set $M$ of its normal words, a certain graph $\Gamma(M)$, and by deriving results on the growth of the algebra in terms of the structure of the graph $\Gamma(M)$, see $[\mathbf{2 2}]$.

A more general conjecture, coming from [1], can be stated as follows: if $R$ is a finitely presented algebra then the growth of $R$ is either subexponential or $R$ contains a free noncommutative subalgebra. This problem, as well as related questions concerning free subalgebras of division rings, have attracted a lot of attention, see [4] for example. Most notably, a conjecture formulated independently by Makar-Limanov and Stafford says that a division algebra $D$ either contains a free noncommutative subalgebra over its center or it is a locally PI-algebra.

Another example of a growth alternative result was given by Okniński and Salwa in the context of the semigroup version of the Tits alternative, [16]. They proved that a finitely generated subsemigroup $S$ of the linear group $G L_{n}(\mathrm{~K})$ over a field $\mathrm{K}$ either contains a free subsemigroup of rank two, or generates an almost-nilpotent subgroup. The second condition yields that the Gelfand-Kirillov dimension of the semigroup algebra $\mathrm{K}[S]$ must be an integer.

\section{Definitions and the necessary background}

Let $A$ be a finitely generated algebra over a field $\mathrm{K}$ and let $V$ be an arbitrary finite dimensional subspace that generates the algebra $A$. Recall that the Gelfand-Kirillov dimension of $A$ is defined by

$$
\operatorname{GKdim}(A)=\lim \sup \left(\log _{n}\left(d_{V}(n)\right)\right)
$$


where $d_{V}(n)=\operatorname{dim}_{K}\left(V^{0}+V^{1}+\cdots+V^{n}\right)$ is the growth function associated with $V$ and $V^{k}=\operatorname{lin}_{\mathrm{K}}\left\{v_{1} \cdots v_{k} \mid v_{i} \in V, 1 \leq i \leq k\right\}$. In the case $S$ is a finitely generated monoid with a generating set $Z$, the growth function of the semigroup algebra $\mathrm{K}[S]$ can be computed by calculating the growth function $d_{S}(n)$ of $S$, namely $d_{S}(n)=\mid\{w \in S \mid w=$ $y_{1} \cdots y_{k}$, for some $\left.y_{i} \in Z, k \leq n\right\} \mid$.

Let $X$ be a finite set and let $F$ denote the set of all words in the alphabet $X$ (including the empty word $\epsilon$, which is identified with the unity 1 of the free algebra $\mathrm{K}\langle X\rangle)$. For every $x \in X$ and $w \in F$ by $\operatorname{deg}_{x} w$ we mean the degree of the word $w$ in $x$. By $|w|$ we denote the length of the word $w$. The support of the word $w$, denoted by $\operatorname{supp}(w)$, stands for the set of all $x \in X$ such that $\operatorname{deg}_{x} w>0$. By $\operatorname{pref}_{n}(w)$, $\operatorname{suff}_{n}(w)$ we denote respectively the prefix and the suffix of the word $w$ of length $n$.

We say that the word $w=x_{1} \cdots x_{r} \in F$ is a subword of the word $v \in$ $F$, where $x_{i} \in X$, if $v=v_{1} x_{1} \cdots v_{r} x_{r} v_{r+1}$, for some $v_{1}, \ldots, v_{r+1} \in F$. If the length $\left|v_{1} x_{1} \cdots v_{r} x_{r}\right|$ is minimal possible then we say that this is the first occurrence of $w$ in $v$. If $v_{2}, \ldots, v_{r}$ are trivial words, then we say that $w$ is a factor of $v$. By $l_{n}(w)=x_{n}$ we denote the $n$-th element of the set $X$ appearing in the word $w$.

Assume that the set $X$ is well ordered. Then on the set $F$ there exists an induced degree-lexicographic order $\leq$. To every element $x \in \mathrm{K}[F]$ one can associate its leading term $\bar{x}$ with respect to $\leq$. For every subset $R \subseteq \mathrm{K}[F]$ by $\alpha(R)$ we denote the set of all leading terms of elements of $R$. If $I$ is an ideal of the free algebra $\mathrm{K}[F]$ then a word $w \in F$ is called normal (modulo $I$ ), if $w$ is not a leading term of any element of $I$.

Describing the normal words of the algebra of the form $\mathrm{K}\langle X\rangle / I$ is related to finding the reduced Gröbner basis of the ideal $I$. The socalled diamond lemma is often used in this context. We will follow the approach and terminology of [5].

We will also fix notation and terminology used in the context of HeckeKiselman monoids. Consider an oriented digraph $\Theta=(V(\Theta), E(\Theta))$. Equalities (i)-(iv) presented in Section 1 are simply called the edge relations. Let $w$ be a word in the free monoid $\langle V(\Theta)\rangle$ on the set $V(\Theta)$. By $[w]$ we denote the equivalence class of the word $w$ with respect to $\mathrm{HK}_{\Theta}$. Also, we define two relations on the monoid $\langle V(\Theta)\rangle$, coming from the graph $\Theta$ : for $w, w^{\prime} \in\langle V(\Theta)\rangle$ we write $w \sim w^{\prime}$ if $[w]=\left[w^{\prime}\right]$, and $w \approx w^{\prime}$ if $w=x y z$, $w^{\prime}=x y^{\prime} z$, where $x, y, y^{\prime}, z \in X$ and $y=y^{\prime}$ is an edge relation in $\mathrm{HK}_{\Theta}$.

An element $v \in V(\Theta)$ is called a source vertex, if $v$ is the beginning of every edge incident to $v$ in $\Theta$. Dually, one defines a sink vertex. 


\section{Hecke-Kiselman monoids defined by a cycle}

In what follows, $\Theta$ will denote an oriented graph. According to Theorem 3 in [13] and Lemma 2.6 in [2], we know that $\operatorname{GKdim}\left(A_{\Theta}\right)=0$ if and only if $\Theta$ is acyclic. Consequently, we start with the case when $\Theta$ is a cycle of length 3 .

Example 1. Consider the graph $\Theta$ of the form:

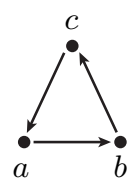

Then $\operatorname{GKdim}\left(A_{\Theta}\right)=1$.

Proof: The monoid $\mathrm{HK}_{\Theta}$ is generated by the idempotents $a, b, c$ subject to the relations: $a b a=b a b=a b, a c a=c a c=c a, b c b=c b c=b c$. It is not hard to see that every word of the free monoid on $a, b, c$ can be rewritten to an equivalent word in $\mathrm{HK}_{\Theta}$ that is a finite factor of one of the following infinite words $(a b c)^{\infty},(a c b)^{\infty}$. Thus are no more than 6 words of length $n$ in $\mathrm{HK}_{\Theta}$, for all $n$. Clearly, this implies that $\operatorname{GKdim}\left(A_{\Theta}\right)=1$.

Our next step is to consider the case of a cyclic oriented graph $\Theta$ of an arbitrary length.

Example 2. Consider the graph $\Theta_{n}$ with $n$ vertices $a_{1}, \ldots, a_{n}$, where $n \geq 4$, that is an oriented cycle:

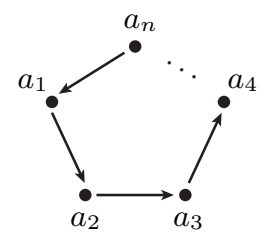

Then $\operatorname{GKdim}\left(A_{\Theta_{n}}\right)=1$.

Proof: Let $F=\langle X\rangle$ be the free monoid with the set $X$ of free generators $a_{1}, \ldots, a_{n}$. We will proceed by induction on the number of vertices $n$. The following observation is crucial.

Lemma 4. Suppose that the cycle $\Theta_{n-1}$ consists of $n-1$ vertices $b_{1}, b_{2}$, $\ldots, b_{n-1}$. Consider an epimorphism $\phi$ from the free monoid $\left\langle b_{1}, \ldots, b_{n-1}\right\rangle$ to the submonoid $\left\langle a_{2}, \ldots, a_{n-1}, a_{n} a_{1}\right\rangle$ of $F$ defined in the following way:

$$
\phi\left(b_{i}\right)= \begin{cases}a_{i+1}, & \text { for } 1 \leq i \leq n-2, \\ a_{n} a_{1}, & \text { for } i=n-1 .\end{cases}
$$


Then $\phi$ induces a homomorphism $\bar{\phi}: \mathrm{HK}_{\Theta_{n-1}} \longrightarrow \mathrm{HK}_{\Theta_{n}}$ given by the formula $\bar{\phi}([w])=[\phi(w)]$, for every $w \in\left\langle b_{1}, \ldots, b_{n-1}\right\rangle$.

Proof: We only need to prove that $\bar{\phi}$ is well defined. It is sufficient, therefore, to check that for every edge relation $w_{1}=w_{2}$ that is defined by $\Theta_{n-1}$ on the free monoid $\left\langle b_{1}, \ldots, b_{n-1}\right\rangle$ we have $\phi\left(w_{1}\right) \sim \phi\left(w_{2}\right)$, where $\sim$ is the relation defined on $F$ by $\Theta_{n}$. This is trivial, if $b_{n-1} \notin \operatorname{supp}\left(w_{1}\right)$. We verify the remaining cases below:

- $\phi\left(b_{n-1}^{2}\right)=a_{n} a_{1} a_{n} a_{1} \sim a_{n} a_{1} a_{1} \sim a_{n} a_{1}=\phi\left(b_{n-1}\right)$;

- for $i \leq n-1$ and $i \notin\{1, n-2\}$, we have $\phi\left(b_{n-1} b_{i}\right)=a_{n} a_{1} a_{i+1} \sim$ $a_{n} a_{i+1} a_{1} \sim a_{i+1} a_{n} a_{1}=\phi\left(b_{i} b_{n-1}\right)$;

- $\phi\left(b_{n-2} b_{n-1} b_{n-2}\right)=a_{n-1} a_{n} a_{1} a_{n-1} \sim a_{n-1} a_{n} a_{n-1} a_{1} \sim a_{n-1} a_{n} a_{1}=$ $\phi\left(b_{n-2} b_{n-1}\right)$;

- $\phi\left(b_{n-1} b_{n-2} b_{n-1}\right)=a_{n} a_{1} a_{n-1} a_{n} a_{1} \sim a_{n} a_{n-1} a_{1} a_{n} a_{1} \sim a_{n} a_{n-1} a_{n} a_{1} \sim$ $a_{n-1} a_{n} a_{1}=\phi\left(b_{n-2} b_{n-1}\right)$;

- $\phi\left(b_{n-1} b_{1} b_{n-1}\right)=a_{n} a_{1} a_{2} a_{n} a_{1} \sim a_{n} a_{1} a_{n} a_{2} a_{1} \sim a_{n} a_{1} a_{2} a_{1} \sim a_{n} a_{1} a_{2}=$ $\phi\left(b_{n-1} b_{1}\right)$;

- $\phi\left(b_{1} b_{n-1} b_{1}\right)=a_{2} a_{n} a_{1} a_{2} \sim a_{n} a_{2} a_{1} a_{2} \sim a_{n} a_{1} a_{2}=\phi\left(b_{n-1} b_{1}\right)$.

We proceed with the proof. The following order

$$
a_{n}<a_{1}<\cdots<a_{n-1}
$$

on the set of generators induces a degree-lexicographic order $\leq$ on $F$. A word $w \in F$ is called reduced if for every $w^{\prime} \sim w$ we have $w \leq w^{\prime}$. Otherwise, we say that $w$ is a reducible word. Of course, for every word $w \in F$ there exists exactly one reduced word $w^{\prime} \in F$ such that $w \sim w^{\prime}$, which we call the reduced form of $w$.

Let $\Theta_{n}^{\prime}$ be the induced subgraph of $\Theta_{n}$ consisting of the vertices $a_{1}, \ldots, a_{n-1}$, and let $F^{\prime}$ be the set of all reduced forms of the elements of the monoid $\mathrm{HK}_{\Theta_{n}^{\prime}}$ in the free monoid $\left\langle a_{1}, \ldots, a_{n-1}\right\rangle$. This set is finite as $\Theta_{n}^{\prime}$ is acyclic.

Notice that every reduced word $w$ in $F$ belongs to the following set:

$$
F^{\prime} \cup F^{\prime} a_{n} F^{\prime} \cup \bigcup_{r=1}^{\infty} F^{\prime} a_{n} w_{1} a_{n} w_{2} a_{n} \cdots w_{r} a_{n} F^{\prime},
$$

where $w_{j} \in F^{\prime}$, for $1 \leq j \leq r$. We will prove the following five claims regarding the case when a reduced word $w$ is of the form

$$
s \cdot a_{n} w_{1} \cdots a_{n} w_{r} a_{n} \cdot t,
$$

where $w_{j} \neq 1$ and $s, t \in F^{\prime}$. 
(1) Every $w_{j}$ must be in one of the following three forms:

(a) $w_{j}=e:=a_{n-1} a_{n-2} \cdots a_{1}$,

(b) $w_{j}=f:=a_{n-2} a_{n-3} \cdots a_{1} a_{n-1}$,

(c) $w_{j}=a_{k} a_{k-1} \cdots a_{1} y a_{n-1}$, where $k<n-2$ and $y \in\left\langle a_{2}, \ldots, a_{n-2}\right\rangle$.

(2) If $w_{j}=e$, for some $j$, then $w_{1}=w_{2}=\cdots=w_{r}$.

(3) If $w_{j}=f$, for some $j$, then $w_{j}=w_{j+1}=\cdots=w_{r}$.

(4) If $w_{j}, w_{j+1}$ are of the form (c) from (1), then $w_{j+1}=a_{1} y a_{n-1}$, for some $y \in\left\langle a_{2}, \ldots, a_{n-2}\right\rangle$.

(5) If $w_{j}=f$, for some $j$, and $r \geq 2$, then $w_{2}=f$.

If we prove these statements above we will see that the growth function $d(k)$ of $\mathrm{HK}_{\Theta_{n}}$, that counts, for the given $k$, the number of reduced words of length less than, or equal to $k$ in $F$, is linear. If fact, it can be presented as $d_{1}(k)+d_{2}(k)$, where:

- $d_{1}(k)$ is the number of reduced words of length $\leq k$ from the set

$$
F^{\prime} \cup F^{\prime} a_{n}\left\langle e a_{n}\right\rangle F^{\prime} \cup\left(F^{\prime} a_{n} \cup F^{\prime} a_{n} F^{\prime} a_{n}\right)\left\langle f a_{n}\right\rangle F^{\prime},
$$

where $\left\langle e a_{n}\right\rangle$ and $\left\langle f a_{n}\right\rangle$ are the free submonoids of $F$ generated by $e a_{n}, f a_{n}$, respectively;

- $d_{2}(k)$ is the number of reduced words $w$ of length $\leq k$ of the form (3.2), where $r \geq 1$ and $w_{j}$ are, for $j \geq 2$, of the form $a_{1} y_{j} a_{n-1}$, where $y_{j} \in\left\langle a_{2}, \ldots, a_{n-2}\right\rangle$.

Both $d_{1}(k)$ and $d_{2}(k)$ are linear. Since $F^{\prime}$ is a finite set, this is clear in the case of $d_{1}(k)$. To see that $d_{2}(k)$ is linear observe that the factor $w^{\prime}=$ $a_{n} w_{2} a_{n} \cdots a_{n} w_{r}$ of the reduced word $w$ belongs to $\left\langle a_{2}, \ldots, a_{n-1}, a_{n} a_{1}\right\rangle$. Thus, from Lemma 4 we get that there exists a reduced word $v$ of length $\leq k$ in $\left\langle b_{1}, \ldots, b_{n-2}\right\rangle$ such that $\phi([v])=\left[w^{\prime}\right]$. Since $w_{1} \in F^{\prime}$ we can see that $d_{2}(k)$ is bounded by the value $\left|F^{\prime}\right|^{3} \cdot d^{\prime}(k)$, where $d^{\prime}$ is the growth function of $\mathrm{HK}_{\Theta_{n-1}}$. The induction hypothesis yields that $d^{\prime}(k)$ is linear, so $d_{2}(k)$ must be linear as well. This yields that $d(k)$ is linear and $\operatorname{GKdim}\left(A_{\Theta_{n}}\right)=1$.

The proofs of (1)-(5) rely on the following easy observations.

Remark 5. Let $v, w$ be elements of $F$ such that $a_{i-1} \notin \operatorname{supp}(v)$ and $a_{i+1} \notin$ $\operatorname{supp}(w)$, for any $i \in\{1, \ldots, n\}$ (here we write $a_{0}=a_{n}$ and $a_{n+1}=a_{1}$ ). The following relations hold:

$$
a_{i} v a_{i} \sim a_{i} v, \quad a_{i} w a_{i} \sim w a_{i} .
$$

In particular, an element of $F$ that contains a factor of the form $a_{i} v a_{i}$ or $a_{i} w a_{i}$ is not reduced. 
This remark follows easily from the defining relations for the (particular) monoid $\mathrm{HK}_{\Theta_{n}}$, see also [9, Lemma 3.4]. The next one is completely obvious.

Remark 6. A word $w \in F$ with a factor $a_{i} a_{j}$, where $a_{i} a_{j} \sim a_{j} a_{i}$ and $a_{i}>a_{j}$ (with respect to $\left.(3.1)\right)$ is not reduced.

We begin with the proof of (1). Consider a reduced word $a_{n} w a_{n}$, where $a_{n} \notin \operatorname{supp}(w)$. First, notice that $\operatorname{deg}_{a_{1}} w=1$ and $\operatorname{deg}_{a_{n-1}} w=1$. Indeed, if $a_{1} \notin \operatorname{supp}(w)$, then the word $a_{n} w a_{n}$ is not reduced, according to Remark 5. The same argument asserts that if $w$ contains a factor of the form $a_{1} w^{\prime} a_{1}$, where $\operatorname{supp}\left(w^{\prime}\right) \subseteq X \backslash\left\{a_{1}, a_{n}\right\}$, then again it cannot be reduced. Similarly, one shows that $\operatorname{deg}_{a_{n-1}} w=1$. Secondly, from Remark 6 it follows immediately that $\operatorname{suff}_{1}(w) \in\left\{a_{1}, a_{n-1}\right\}$ (otherwise, this element commutes with $a_{n}$ ). Since $|w|>0$, we consider two cases.

Case 1: Let $\operatorname{suff}_{1}(w)=a_{1}$. We prove that $w=e$. We proceed in two steps. First, we prove inductively that $w=v e=v a_{n-1} \cdots a_{1}$, for some $v \in F^{\prime}$, and next we show that $v=1$.

We begin with the induction. Let $w=w^{\prime} a_{1}$, for some $w^{\prime} \in F^{\prime}$. Since $|w| \geq 2$, it follows that $w^{\prime} \neq 1$. Again, from Remark 6 it follows that $\operatorname{suff}_{1}\left(w^{\prime}\right)$ cannot be an element of $X$ that commutes with $a_{1}$ and clearly $\operatorname{suff}_{1}\left(w^{\prime}\right) \notin\left\{a_{1}, a_{n}\right\}$. Consequently, $a_{n} w a_{n}=a_{n} w^{\prime \prime} a_{2} a_{1} a_{n}$, for some $w^{\prime \prime} \in F^{\prime}$ and the first step of induction follows. Assume now that $w=w^{\prime \prime} a_{k} a_{k-1} \cdots a_{1}$ for some $1<k<n-1$. Of course, $w^{\prime \prime} \neq 1$, as otherwise $a_{n-1} \notin \operatorname{supp}\left(a_{n} w a_{n}\right)$, a contradiction. We want to show that $\operatorname{suff}_{1}\left(w^{\prime \prime}\right)=a_{k+1}$. As before, using Remark 6 , we see that $\operatorname{suff}_{1}\left(w^{\prime \prime}\right) \neq$ $a_{q}$, where $q>k+1$. Also, it is clear that $\operatorname{suff}_{1}\left(w^{\prime \prime}\right) \notin\left\{a_{k-1}, a_{k}\right\}$. If $\operatorname{suff}_{1}\left(w^{\prime \prime}\right)=a_{l}$, for $l<k-1$, then the word $a_{n} w a_{n}$ has a factor $a_{l} z a_{l}$, where $a_{l-1} \notin \operatorname{supp}(z)$, so it is not reduced by Remark 5. This shows that $q=k+1$, completing the induction step. We have shown that $w=v e$, for some $v \in F^{\prime}$. However, if we had $v \neq 1$ and $\operatorname{suff}_{1}(v)=a_{i}$, for $1 \leq i \leq n-3$ (otherwise $v$ would contain a reducible factor) then $w$ would contain a factor $a_{i} z^{\prime} a_{i}$ with $a_{i-1} \notin \operatorname{supp}\left(z^{\prime}\right)$, which would make it reducible. Therefore $v=1$, and it follows that $w=e$.

Case 2: Let $\operatorname{suff}_{1}(w)=a_{n-1}$. Since $\operatorname{deg}_{a_{1}} w=\operatorname{deg}_{a_{n-1}} w=1$, there exist $x, y \in F^{\prime}$ such that $a_{n} w a_{n}=a_{n} x a_{1} y a_{n-1} a_{n}$ and $\operatorname{supp}(x), \operatorname{supp}(y) \subseteq$ $X \backslash\left\{a_{1}, a_{n-1}, a_{n}\right\}$. Applying arguments similar to those used in the previous case, we see that $x a_{1}$ is of one of the forms: $a_{k} a_{k-1} \cdots a_{2} a_{1}$, where $1 \leq k \leq n-2$. If $k=n-2$ then clearly $w=f$ as the element $a_{n-2} \cdots a_{2} a_{1} a_{s}$ is reducible, for all $s<n-1$, again by Remark 5 . If $1 \leq k<n-2$, then the element $w$ is exactly of the form (c) listed in (1). 
Statement (1) is verified. We proceed to prove (2). Take a reduced word $w$ of the form (3.2). If $r=1$ there is nothing to prove. Assume that $r \geq 2$. If $k \neq n-1$ then the word $e a_{n} a_{k}$ contains a factor of the form $a_{k} \cdots a_{1} a_{n} a_{k}$ which does not contain $a_{k+1}$. Thus, none of these words may be reduced, due to Remark 5. Similarly, no words of the form $a_{k} a_{n} e$, where $k \neq 1$ may be reduced. In view of (1), this proves the assertion of (2). Similarly, one shows that if $f a_{n} a_{k}$ is reduced then $k=n-2$. Therefore, if $w_{j}=f$, for some $j$, then $w_{j+1}=\cdots=w_{r}=f$, so that (3) holds.

The statement of (4) is also easy to check. Each reduced word of the form $a_{n} w_{j} a_{n} w_{j+1} a_{n}$, as defined in this case, contains a factor $a_{n-1} a_{n} a_{k}$, for $k<n-2$. But unless $k=1$, we have $a_{n-1} a_{n} a_{k} \sim a_{k} a_{n-1} a_{n}$. Moreover $a_{k} a_{n-1} a_{n}<a_{n-1} a_{n} a_{k}$ in the deg-lex order on $F$, defined by (3.1). Since $a_{n} w_{j} a_{n} w_{j+1} a_{n}$ is reduced, such case cannot occur and $w_{j+1}$ must be of the form $a_{1} y a_{n-1}$, where $y \in\left\langle a_{2}, \ldots, a_{n-2}\right\rangle$, as claimed.

Assume that $w_{j}=f$, for some $j$, and choose minimal such $j$. Suppose $j \geq 3$. It follows from (1)-(3) that each of $w_{1}, \ldots, w_{j-1}$ is of the form (c). Hence, by (4) we must have $w_{i}=a_{1} y_{i} a_{n-1}$, for $i=2, \ldots, j-1$, where $y_{i} \in\left\langle a_{2}, \ldots, a_{n-2}\right\rangle$. Suppose $y_{j-1}=x a_{s}$, for some $s$ and some $x \in F$. Then the factor $a_{s} a_{n-1} a_{n} a_{n-2} \cdots a_{s}$ of $a_{n} w_{j-1} a_{n} w_{j} a_{n}$ does not contain $a_{s-1}$, a contradiction (because it must be reduced). It follows that $y_{j-1}$ is the empty word and $w_{j-1}=a_{1} a_{n-1}$. Since $w_{j-2}$ must be of the form (c), we see that $a_{n-1} a_{n} a_{1} a_{n-1}$ is a factor of $w_{j-2} a_{n} w_{j-1}$. Since this is not a reduced word, we get a contradiction. It follows that $j \leq 2$. Hence (5) is proved.

\section{Free submonoids of $\mathrm{HK}_{\Theta}$}

In this section we present a family of examples of graphs $\Theta$, for which the monoid $\mathrm{HK}_{\Theta}$ contains a free submonoid of rank 2. We begin with the case of two cycles of length three connected by a path of length 0 .

Example 3. Let $\Theta$ be a graph with vertices $a, b, c, d$ and $\Psi$ be a graph with vertices $a, b, c, e, f$ of the following form:
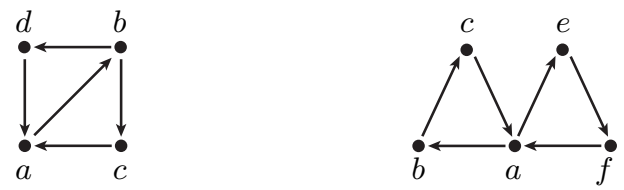

Then the submonoid $\langle[a c b],[a d b]\rangle$ of $\mathrm{HK}_{\Theta}$ and the submonoid $\langle[a c b],[a f e]\rangle$ of $\mathrm{HK}_{\Psi}$ are both free of rank 2 . 
Proof: It is clear that no word of the submonoid $\langle a c b, a d b\rangle$ of the free monoid $F=\langle a, b, c, d\rangle$ can be rewritten in $F$ in any way using the edge relations defining the monoid $\mathrm{HK}_{\Theta}$ except by the idempotent relations of type $x=x x$, for $x \in\{a, b, c, d\}$. The same argument holds for the submonoid $\langle a c b, a f e\rangle$ of the free monoid $F^{\prime}=\langle a, b, c, e, f\rangle$.

We will now consider the case of two disjoint cycles connected by a path of a nonzero length. The following definition will be useful.

Definition 7. Let $\Theta$ be a simple oriented digraph and let $F=\langle V(\Theta)\rangle$ a free monoid generated by the set of vertices of $\Theta$. Suppose that a cycle $\Theta^{\prime}$ of length $n$ of the form $x_{1} \rightarrow x_{2} \rightarrow \cdots \rightarrow x_{n} \rightarrow x_{1}$ is a subgraph of $\Theta$. We will say that the word $w \in F$ is $\Theta^{\prime}$-free if the maximal subword $v$ of $w$ with $\operatorname{supp}(v) \subseteq V\left(\Theta^{\prime}\right)$ is a factor of the infinite word $\left(x_{n} x_{n-1} \cdots x_{1}\right)^{\infty}$.

Example 4. Let $\Theta$ be a graph of the form:

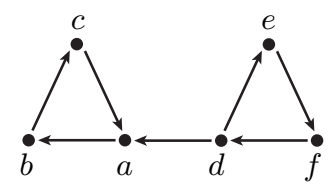

Then the submonoid $\langle[a c b],[f e d]\rangle$ of $\mathrm{HK}_{\Theta}$ is free.

Proof: Let $F=\langle X\rangle$ be the free monoid on the set $X=\{a, b, c, d, e, f\}$ and let $H=\langle a c b, f e d\rangle$ be a submonoid of $F$. We define a subset $W$ of $F$ as follows: an element $w$ belongs to $W$ if and only if the following three conditions are satisfied:

(1) $\operatorname{supp}(w)=X$;

(2) the first appearance of $a$ in $w$ is earlier then the ones of $b$ and $c$, and the first appearance of $f$ in $w$ is earlier then the ones of $d$ and $e$;

(3) every word in $W$ is both $\{a, b, c\}$-free and $\{d, e, f\}$-free.

The outline of the proof is as follows. First, we prove that $W$ is closed under equivalence classes of $F$ under the relation $\sim$ defined for $\Theta$ in Section 2. Then, to each element $v \in W$ we assign an infinite sequence $N_{v}$ of nonnegative integers $\left(i_{1}, j_{1}, i_{2}, j_{2}, \ldots\right)$ such that $N_{v}=N_{v^{\prime}}$ if $v \sim v^{\prime}$. It is clear that $H$ is a subset of $W$ and it will be straightforward to verify that $N_{w} \neq N_{w^{\prime}}$, for any two different members $w, w^{\prime}$ of $H$. From this we will easily deduce that $\langle[a c b],[f e d]\rangle$ is a free submonoid of rank 2 in $\mathrm{HK}_{\Theta}$.

Take any $w \in W$ and $w^{\prime} \in F$ such that $w^{\prime} \sim w$. We claim that $w^{\prime} \in W$. Clearly, it is enough to confirm this in the case when $w$ and $w^{\prime}$ 
Satisfy $w \approx w^{\prime}$, as the relation $\sim$ is the transitive closure of $\approx$ defined in Section 2. Thus, we may assume that $w=u_{1} t u_{2}$ and $w^{\prime}=u_{1} t^{\prime} u_{2}$, for some $u_{1}, u_{2} \in F$ and an edge relation $t=t^{\prime}$ in $\mathrm{HK}_{\Theta}$. Since $w \in F$, we only need to consider the following cases:

(a) $\left\{t, t^{\prime}\right\} \in\{x, x x\}, x \in X$;

(b) $\left\{t, t^{\prime}\right\} \in\{x y, y x\}$, where $x y \approx y x$, and $x, y \in X$;

(c) $\left\{t, t^{\prime}\right\} \subseteq\{d a, a d a, d a d\}$.

Indeed, the case $\left\{t, t^{\prime}\right\} \subseteq\{x y, x y x, y x y\}$, where $x y \neq d a$ is excluded according to condition (3) in the definition of $W$. Now it is basically clear that $w^{\prime}$ satisfies conditions (1)-(3) and thus it belongs to $W$.

We will now introduce a preparatory notation in order to associate a sequence $N_{w}$ to every $w \in W$, as mentioned in the outline of the proof. From (1)-(3) it follows that $w$ can be represented in two forms. First

$$
w=u_{1} a_{1} u_{2} c_{1} u_{3} b_{1} u_{4} a_{2} u_{5} c_{2} u_{6} b_{2} \cdots,
$$

where $a_{i_{1}}, b_{i_{2}}, c_{i_{3}}, u_{i_{4}}$ are trivial for sufficiently large $i_{1}, i_{2}, i_{3}, i_{4}$ and $u_{i} \in\langle d, e, f\rangle, a_{i} \in\langle a, d, e, f\rangle, b_{i} \in\langle b, d, e, f\rangle, c_{i} \in\langle c, d, e, f\rangle$. Moreover, the first and last letter of every $a_{i}, b_{i}, c_{i}$ is equal to $a, b, c$, respectively.

The second form of $w$ is as follows:

$$
w=v_{1} f_{1} v_{2} e_{1} v_{3} d_{1} v_{4} f_{2} v_{5} e_{2} v_{6} d_{2} \cdots
$$

where again $d_{j_{1}}, e_{j_{2}}, f_{j_{3}}, v_{j_{4}}$ are trivial for sufficiently large $j_{1}, j_{2}, j_{3}, j_{4}$ and $v_{j} \in\langle a, b, c\rangle, d_{j} \in\langle a, b, c, d\rangle, e_{j} \in\langle a, b, c, e\rangle, f_{j} \in\langle a, b, c, f\rangle$. Moreover, the first and last letter of $d_{j}, e_{j}, f_{j}$ is equal to $d, e, f$, respectively. It is clear that if any member of $F$ (not necessarily from $W$ ) is of the form (4.1) or (4.2) then this form is uniquely determined.

We also need certain numerical invariants of elements of $W$ to proceed. For each factor $v$ of $w \in F$ we define positive integers $s_{w}(v), t_{w}(v)$. The first one is the position of the first letter of $v$ in $w$, in other words: $l_{s_{w}(v)}(w)=l_{1}(v)$, according to the notation adopted in Section 2. The number $t_{w}(v)$ denotes the position of the last letter of $v$ in $w$, which means that $l_{t_{w}(v)}(w)=l_{|v|}(v)$.

The sequence $N_{w}$ is defined by the recurrence in which the consecutive elements $i_{1}, j_{1}, i_{2}, j_{2}, \ldots$ are defined. If at any step one of the numbers $i_{l}$ or $j_{l}$ turns out to be zero, for $l>1$, then the recurrence terminates and all succeeding elements of $N_{w}$ are, by definition, equal to zero. 
The first step depends on which of the two numbers $s_{w}\left(d_{1}\right)$ and $t_{w}\left(a_{1}\right)$ is greater.

Case 1: Let $s_{w}\left(d_{1}\right)<t_{w}\left(a_{1}\right)$. Then we put $i_{1}=0$ and $j_{1}=r$, where $r>0$ is the largest integer such that $s_{w}\left(d_{r}\right)<t_{w}\left(a_{1}\right)$.

Case 2: Let $s_{w}\left(d_{1}\right)>t_{w}\left(a_{1}\right)$. Let $k>0$ be the largest integer such that $t_{w}\left(a_{k}\right)<s_{w}\left(d_{1}\right)$. Then:

- If the word $(a c b)^{k}$ is not a subword of $w$, then we put $i_{1}=k-1$, and we define $j_{1}$ as the maximal $s$ such that $(f e d)^{s}$ is a subword of $w$. Moreover, we put $i_{2}=0$, which (according to the above rules) terminates the entire recurrence.

- If the word $(a c b)^{k}$ is a subword of $w$, then we put $i_{1}=k$. Moreover, we also define $j_{1}$. If $a_{i_{1}+1}$ is not trivial, then $j_{1}=r$, where $r$ is the largest number such that $s_{w}\left(d_{r}\right)<t_{w}\left(a_{i_{1}+1}\right)$. In the opposite case $j_{1}$ is defined as a maximal $m$ such that $(f e d)^{m}$ is a subword of $w$.

Note that conditions (1)-(3) of the definition of $W$ assert that $j_{1}>0$, no matter the case considered. Thus, if the recurrence has not already terminated, we define the steps for $l>0$ in the following manner.

The defining step for $i_{l+1}$ (this assumes that in the previous step we have established $j_{l}>0$ ). If $d_{j_{l}+1}$ is trivial, then $i_{l+1}=r-\sum_{m=1}^{l} i_{m}$, where $r$ is the greatest integer such that $(a c b)^{r}$ is a subword of $w$. If $d_{j_{l}+1} \neq 1$, then let $k$ be the maximal integer such that $t_{w}\left(a_{k}\right)<$ $s_{w}\left(d_{j_{l}+1}\right)$. Then if $(a c b)^{k}$ is not a subword of $w$, we put $i_{l+1}=k-$ $\sum_{m=1}^{l} i_{m}-1$ and $j_{l+1}=0$. If, however, $(a c b)^{k}$ is a subword $w$, then we put $i_{l+1}=k-\sum_{m=1}^{l} i_{m}>0$.

The defining step for $j_{l+1}$ (assuming that $i_{l+1} \neq 0$ ). If $a_{i_{l+1}+1}$ is not trivial, then $j_{l+1}$ is defined as $r-\sum_{m=1}^{l} j_{m}$, where $r$ is the greatest integer such that $s_{w}\left(d_{r}\right)<t_{w}\left(a_{i_{l+1}+1}\right)$. In the opposite case, $j_{l+1}$ is defined as $p-\sum_{m=1}^{l} j_{m}$, where $p$ is the maximal integer such that $(f e d)^{p}$ is a subword of $w$.

We will now show that the condition $w \approx w^{\prime} \in W$ implies that the sequences $N_{w}=\left(i_{1}, j_{1}, \ldots\right)$ and $N_{w^{\prime}}=\left(i_{1}^{\prime}, j_{1}^{\prime}, \ldots\right)$ are equal. In other words, we assume that $w=u t v$ and $w^{\prime}=u t^{\prime} v$, where $v, w \in F$ and $t$, $t^{\prime}$ are as in one of the cases (a), (b), (c) listed above. Write $w^{\prime}$ in the forms (4.1) and (4.2):

$$
\begin{aligned}
& w^{\prime}=u_{1}^{\prime} a_{1}^{\prime} u_{2}^{\prime} c_{1}^{\prime} u_{3}^{\prime} b_{1}^{\prime} u_{4}^{\prime} a_{2}^{\prime} u_{5}^{\prime} \cdots, \\
& w^{\prime}=v_{1}^{\prime} f_{1}^{\prime} v_{2}^{\prime} e_{1}^{\prime} v_{3}^{\prime} d_{1}^{\prime} v_{4}^{\prime} f_{2}^{\prime} v_{5}^{\prime} \cdots,
\end{aligned}
$$


with the appropriate conditions on $a_{i}^{\prime}, b_{i}^{\prime}, c_{i}^{\prime}, d_{i}^{\prime}, e_{i}^{\prime}, f_{i}^{\prime}, u_{i}^{\prime}, v_{i}^{\prime}$. We will confirm three observations:

(i) If in the form (4.1) of $w$ we have $a_{i} \neq 1\left(b_{i} \neq 1, c_{i} \neq 1\right.$ respectively), then $a_{i}^{\prime} \neq 1\left(b_{i}^{\prime} \neq 1, c_{i}^{\prime} \neq 1\right.$ respectively) in (4.3).

(ii) If in the form (4.2) of $w$ have $d_{i} \neq 1\left(e_{i} \neq 1, f_{i} \neq 1\right.$ respectively), then $d_{i}^{\prime} \neq 1\left(e_{i}^{\prime} \neq 1, f_{i} \neq 1\right.$ respectively) in (4.4).

(iii) $s_{w}\left(d_{i}\right)<t_{w}\left(a_{j}\right) \Leftrightarrow s_{w^{\prime}}\left(d_{i}^{\prime}\right)<t_{w^{\prime}}\left(a_{j}^{\prime}\right)$, for all $i, j$.

It clearly follows from (i)-(iii) that the entire recurrence construction of $N_{w}$ can be applied to $w^{\prime}$ leading to $N_{w}=N_{w^{\prime}}$, as desired.

Consider the set $L(w)$ of all nontrivial factors $\left\{a_{i}, b_{i}, c_{i}, u_{i}\right\}$ of (4.1), for $i=1,2, \ldots$ To simplify notation we assume that if $z$ is the $n$-th element of $L(w)$ (by reading $w$ from the left), then $z^{\prime}$ is the $n$-th element of the corresponding set of factors $L\left(w^{\prime}\right)$ of (4.3). By the symmetry of (i)-(iii) we may assume that $t<t^{\prime}$ in the deg-lex order on $F$.

Assume that $\left\{t, t^{\prime}\right\}$ is as in (a), namely $t=x$, for $x \in X$. Since $t$ is in the support of some $z \in L(w)$, then $t^{\prime}=x x$ is a factor of $z^{\prime} \in L\left(w^{\prime}\right)$. Moreover $y=y^{\prime}$, for all $z \neq y \in L(w)$. Thus (i) follows. By the dual argument applied to the factor set of (4.2) we verify (ii). And clearly, (iii) is satisfied. Indeed, if $s_{w}\left(d_{i}\right), t_{w}\left(a_{j}\right)$ are less than or equal to $s_{w}(t)$, then their order cannot change when we pass to $w^{\prime}$. In the opposite case both numbers increase by one, while we pass to $w^{\prime}$.

If $\left\{t, t^{\prime}\right\}$ is as in (b), we have $t=x y$, where $x \in\{a, b, c\}$ and $y \in$ $\{d, e, f\}$, excluding the case $x y=a d$. To verify (i) we need to consider two possibilities. First: $t$ is a factor, but not a suffix of $x_{i}$, for some $i$. Second: there exist $i, k$ such that $x$ is a suffix of $x_{i} \in L(w)$ and $y$ is a prefix of $u_{k}$. In both cases $t^{\prime}=y x$ is a factor of $x_{i}^{\prime} \in L\left(w^{\prime}\right)$, while it is possible that $u_{k}^{\prime}$ is trivial. However, all elements of $L(w)$ except $x_{i}$ and possibly $u_{k}$, remain unchanged when we pass to $w^{\prime}$. So (i) must hold. By a dual argument one can verify (ii). Again, (iii) easily follows. If $x, y \notin\{a, d\}$ then the numbers $s_{w}\left(d_{i}\right), t_{w}\left(a_{j}\right)$ remain unchanged while passing to $w^{\prime}$. And if either $x \in\{a, d\}$, or $y \in\{a, d\}$, only one of these numbers can change by 1 , since $x y \neq a d$.

In case $(c)$, when $t=d a$ or $a d a$ we will only prove (i) and (iii). Again, (ii) follows from dual arguments. We can see that $t$ must be a factor of some factor $u_{k} a_{i} u_{k+1}$ of $w$, that appears in (4.1). Since $u_{k}, u_{k+1} \in\langle d, e, f\rangle$ we have two possible cases:

- $u_{k} a_{i}=(u \underbrace{d)(a v}_{t})$, for some $u, v \in F$.

- $t$ is a factor of $a_{i}$. 
Consider the first case. Here, we have $t=d a, u_{k}=u d, a_{i}=a v$, for some $u, v \in F$ and the following three configurations are possible:

- $u_{k}^{\prime} a_{i}^{\prime}=(u)(\underbrace{a d a}_{t^{\prime}} v)$ and $x^{\prime}=x$, for $x \in L(w)$, where $x \neq a_{i}, u_{k}$.

- $u_{k}^{\prime} a_{i}^{\prime}=(\underbrace{d)(a d}_{t^{\prime}} v)$ and $x^{\prime}=x$, for $x \in L(w)$, where $x \neq a_{i}, u_{k}$ (when $v \neq 1$ ).

- $u_{k}^{\prime} a_{i}^{\prime} u_{k+1}^{\prime}=(u \underbrace{d)(a)(d}_{t^{\prime}} u_{k+1})$ and $x^{\prime}=x$, for $x \in L(w)$, where $x \neq a_{i}, u_{k}, u_{k+1}($ when $v=1)$.

In the second case, when $t$ is a factor of $a_{i}$ we have yet another five possible configurations:

- If $a_{i}=u t v$, where $u, v \in F$ and $u, v \neq 1$, then $a_{i}^{\prime}=u t^{\prime} v$ and $x^{\prime}=x$, for $x \in L(w), x \neq a_{i}$.

- If $a_{i}=t v$, where $v \in F, v \neq 1$, then $t$ must be equal to ada and we have $u_{k}^{\prime} a_{i}^{\prime}=(u_{k} \underbrace{d)(a d}_{t^{\prime}} v)$ and $x^{\prime}=x$, for $x \in L(w), x \neq a_{i}, u_{k}$.

- If $a_{i}=u t$, where $u \in F, u \neq 1$, then we either have:

$-a_{i}^{\prime}=u \underbrace{\operatorname{ada}}_{t^{\prime}}$ and $x^{\prime}=x$ for $x \in L(w), x \neq a_{i}$, or

$-a_{i}^{\prime} u_{k+1}^{\prime}=(u \underbrace{d a)(d}_{t^{\prime}} u_{k})$ and $x^{\prime}=x$, for $x \in L(w), x \neq a_{i}, u_{k+1}$.

- If $a_{i}=t$, then $t=a d a$ and we have $u_{k}^{\prime} a_{i}^{\prime} u_{k+1}^{\prime}=(u_{k} \underbrace{d)(a)(d}_{t^{\prime}} u_{k+1})$, and $x^{\prime}=x$, for $x \in L(w), x \neq a_{i}, u_{k}, u_{k+1}$.

This gives a total of eight configurations (in both cases) and it is clear that (i) holds in each of them. Let us verify (iii). From the definition of $W$ it follows that the elements $a$ in the factors $t$ and $t^{\prime}$ of $w, w^{\prime}$ appear in the same respective factors $a_{i}$ and $a_{i}^{\prime}$ of (4.1) and (4.3). The same holds for elements $d$ in the factors of (4.2), (4.4). Consequently, for all pairs $(n, m)$ from the set $N:=\left\{\left(s_{w}\left(d_{p}\right), t_{w}\left(a_{q}\right)\right), p, q=1,2, \ldots\right\}$ such that $n, m<\min \left\{t_{w}\left(a_{i}\right), s_{w}\left(d_{j}\right)\right\}$ and $n, m>\max \left\{t_{w}\left(a_{i}\right), s_{w}\left(d_{j}\right)\right\}$ the inequality between of $n$ and $m$ does not change while we pass to $w^{\prime}$. Going though the configurations listed above we can easily verify (iii) for the remaining pairs of $N$.

As a result, we have shown that for two elements $w \approx w^{\prime}$ from $W$ we have $N_{w}=N_{w^{\prime}}$. Thus the equality between these sequences occur also in the case when $w \sim w^{\prime}$. Now we apply this fact to see that $\langle[a c b],[f e d]\rangle$ is a free submonoid of $\mathrm{HK}_{\Theta}$. 
Let $w=(a c b)^{m_{1}}(f e d)^{n_{1}}(a c b)^{m_{2}}(f e d)^{n_{2}} \ldots$ belong to the monoid $H$ for some nonnegative integers $\bar{w}=\left(m_{1}, n_{1}, m_{2}, n_{2}, \ldots\right)$. Here we assume that either we have $m_{1}>0, n_{1}>0$, or $m_{1}=0, n_{1}, m_{2}>0$ and that apart from these first few elements, the succeeding members of $\bar{w}$ are positive, up to some point, after which all of them become zeros. By carefully applying the definition of $N_{w}$, it is easy to that $N_{w}=\bar{w}$. Moreover, it is clear that if $w, w^{\prime} \in\langle a c b, f e d\rangle$ and $w \sim w^{\prime}$, then $\widetilde{w}=$ $(a c b)(f e d) w \sim(a c b)(f e d) w^{\prime}=\widetilde{w^{\prime}}$ and $\widetilde{w}, \widetilde{w^{\prime}} \in W$. Hence $N_{\widetilde{w}}=N_{\widetilde{w^{\prime}}}$, which easily implies that $N_{w}=N_{w^{\prime}}$. Hence, the images of all elements of the monoid $\langle a c b, f e d\rangle$ under the natural homomorphism $F \longrightarrow \mathrm{HK}_{\Theta}$ are pairwise distinct. The assertion of Example 4 follows.

We continue with a generalization that is crucial for the proof of Theorem 1.

Example 5. Consider $\Theta_{n, k, m}$ to be a graph with $n+k+m$ vertices that consists of: a cycle $\Phi_{n}$ of length $n \geq 3$ of the form $a_{1} \rightarrow a_{2} \rightarrow \cdots \rightarrow$ $a_{n} \rightarrow a_{1}$, a cycle $\Psi_{m}$ of length $m \geq 3$ of the form $b_{1} \rightarrow b_{2} \rightarrow \cdots \rightarrow$ $b_{m-1} \rightarrow b_{m} \rightarrow b_{1}$, and a path of length $k+1$ from $b_{1}$ to $a_{1}$, for $k \geq 0$. For $k=0$ this means that we have $b_{1} \rightarrow a_{1}$, and for $k>0$ the path is of the form $b_{1} \rightarrow z_{1} \rightarrow \cdots \rightarrow z_{k} \rightarrow a_{1}$ as in the picture below:

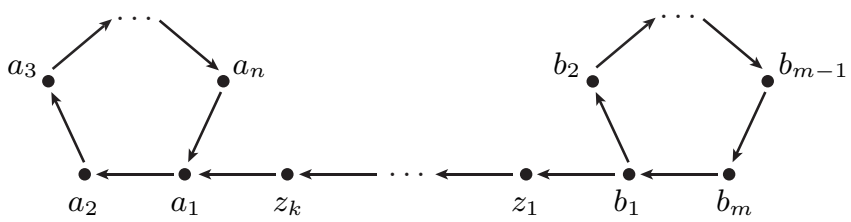

Then the submonoid $\left\langle\left[a_{1} a_{n} \cdots a_{2}\right],\left[b_{m} b_{m-1} \cdots b_{1} z_{1} \cdots z_{k}\right]\right\rangle$ of the monoid $\mathrm{HK}_{\Theta n, k, m}$ is free.

Proof: We will first consider the case when $\Theta_{3, k, 3}:=\Theta_{k}$ is a graph of $6+k$ vertices and consists of two cycles $\{a, b, c\},\{d, e, f\}$ joined by a path of length $k+1$, for $k \geq 0$, as shown in the picture (for $k>0$ ):

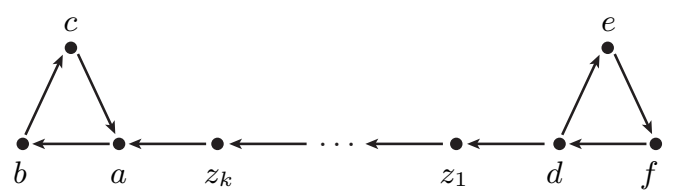

Let us see that $\mathrm{HK}_{\Theta_{k}}$ has a free submonoid of rank 2 generated by [acb] and $\left[\right.$ fed $\left.z_{1} \cdots z_{k}\right]$.

For each $k \geq 0$ consider the set $X_{k}=\left\{a, b, c, d, e, f, z_{1}, \ldots, z_{k}\right\}$ (when $k=0$ this is just $\{a, b, c, d, e, f\}$ ). Let $F_{k}=\left\langle X_{k}\right\rangle$ be a free monoid on the 
set $X_{k}$. By $\sim_{k}, \approx_{k}$ we denote two relations on $F_{k}$ defined in Section 2 for $\Theta_{k}$. We proceed by induction on $k$. The first step has already been verified in Example 4. Let us then assume that the assertion holds for $\Theta_{k}$, where $k \geq 0$.

Consider the subset $W_{k+1}$ of $F_{k+1}$ that consists of all words that are both $\{a, b, c\}$-free and $\{d, e, f\}$-free. As in the previous example it is not difficult to show that $W_{k+1}$ is closed under equivalence classes of $\sim_{k+1}$ in $F_{k+1}$. If $w=r s t$ and $v=r s^{\prime} t$, for $w \in W_{k+1}, v \in F_{k+1}$, and $s=s^{\prime}$ is an edge relation in $\mathrm{HK}_{\Theta_{k+1}}$ then the order of appearance of elements from sets $\{a, b, c\}$ and $\{d, e, f\}$ cannot change while we pass from $w$ to $v$. The possible edge relations are listed below:

(a) $\left\{s, s^{\prime}\right\}=\{x, x x\}$, where $x \in X_{k+1}$.

(b) $\left\{s, s^{\prime}\right\}=\left\{x y, y x \mid x \in\left\{a, b, c, z_{1}, \ldots, z_{k}\right\}, y \in\left\{d, e, f, z_{1}, \ldots, z_{k}\right\}\right\}$ except for the cases when $x=y$ and when we have an arrow $x \rightarrow y$ in $\Theta_{k+1}$.

(c) $\left\{s, s^{\prime}\right\}=\left\{x z_{k+1}, z_{k+1} x \mid x \in X_{k+1} \backslash\left\{a, z_{k}\right\}\right\}$.

(d) $\left\{s, s^{\prime}\right\} \subseteq\left\{x y, x y x, y x y \mid x=z_{i}, y=z_{i+1}\right.$ for $\left.1 \leq i \leq k-1\right\}$ and $\left\{s, s^{\prime}\right\} \subseteq\left\{d z_{1}, d z_{1} d, z_{1} d z_{1}\right\}$.

(e) $\left\{s, s^{\prime}\right\} \subseteq\left\{z_{k} z_{k+1}, z_{k} z_{k+1} z_{k}, z_{k+1} z_{k} z_{k+1}\right\}$.

(f) $\left\{s, s^{\prime}\right\} \subseteq\left\{z_{k+1} a, z_{k+1} a z_{k+1}, a z_{k+1} a\right\}$.

Let $\overleftarrow{w} \in F_{k+1}$ arise from the word $w \in F_{k+1}$ by applying all possible commutation edge relations (that is, of types (b), (c) above) of $\mathrm{HK}_{\Theta_{k+1}}$ in order to move all elements $a, b, c$ as far to the left as possible. This is obviously well defined. We also define an element $\overleftarrow{w}_{z_{k+1}}$ in $F_{k}$, for every $w \in F_{k+1}$, as the maximal subword $v$ of $\overleftarrow{w}$ such that $\operatorname{deg}_{z_{k+1}} v=0$ To simplify notation put $z:=z_{k+1}$. Consider a map $\phi_{k+1}:\left[W_{k+1}\right] \longrightarrow$ $\mathrm{HK}_{\Theta_{n}}$, that associates to the class $[w]$ of an element $w \in W_{k+1}$ the class of the element $\overleftarrow{w}_{z}$. We will show that $\phi_{k+1}$ is a function. This clearly amounts to showing that

$$
w \approx_{k+1} v \Rightarrow \overleftarrow{w}_{z} \sim_{k} \overleftarrow{v}_{z}
$$

Consider the cases (a)-(f) listed above. The implication in (4.5) is obvious in cases (b) and (d), as we have $\overleftarrow{w}=\overleftarrow{v}$ and thus $\overleftarrow{w}_{z}=\overleftarrow{v}_{z}$ Also in case (c) it is clear that $\overleftarrow{w}_{z}=\overleftarrow{v}_{z}$. The remaining cases require more detailed explanation.

In case (a) we have $w=r x t$ and $v=r x x t$, for some $x \in X_{k+1}$. When $x=z$ it is clear that $\overleftarrow{w}_{z}=\overleftarrow{v}_{z}$. If $x \neq z$ then $\overleftarrow{w}=r^{\prime} x t^{\prime}$ and $\overleftarrow{v}=r^{\prime} x x t^{\prime}$ for some $r^{\prime}, t^{\prime} \in F_{k+1}$. Thus $\overleftarrow{w}_{z} \approx_{k} \overleftarrow{v}_{z}$

In case (e) the following three subcases arise: 
- Let $w=r z_{k} z t, v=r z z_{k} z t$. Then clearly $\overleftarrow{w} \approx_{k+1} \overleftarrow{v}$ with the edge relation $z_{k} z=z z_{k} z$, hence $\overleftarrow{w}_{z}=\overleftarrow{v}_{z}$

- Let $w=r z_{k} z t, v=r z_{k} z z_{k} t$. Then $\overleftarrow{w}=r^{\prime} z_{k} z a^{n} t^{\prime}, \overleftarrow{v}=r^{\prime} z_{k} z a^{n} z_{k} t^{\prime}$ for some $n \geq 0$ and $r^{\prime}, t^{\prime} \in F_{k+1}$. Since we have $z_{k} a^{n} \sim_{k} z_{k} a^{n} z_{k}$ then $\overleftarrow{w}_{z} \sim_{k} \overleftarrow{v}_{z}$

- Finally, if $w=r z z_{k} z t$ and $v=r z_{k} z z_{k} t$, then we proceed analogously as in the previous subcase.

In case (f) we also consider three subcases:

- Let $w=r z a t, v=r z a z t$. Then $\overleftarrow{w}=\overleftarrow{r} z a a^{n} t^{\prime}$ and $\overleftarrow{v}=\overleftarrow{r} z a a^{n} z t^{\prime}$ with $\overleftarrow{t}=a^{n} t^{\prime}$, for some $t^{\prime} \in F_{k+1}$ and $n \geq 0$. Hence $\overleftarrow{w}_{z} \sim_{k} \overleftarrow{v}_{z}$

- Let $w=r z a t, v=$ razat. Then $\overleftarrow{w}_{z}=\overleftarrow{r}_{z} a \overleftarrow{t}_{z}$ and $\overleftarrow{v}_{z}=(\overleftarrow{r a})_{z} a \overleftarrow{t}_{z}$ Notice that $(\overleftarrow{r a})_{z}=p a q$ and $\overleftarrow{r}=p q$, for some $p \in F_{k+1}, q \in$ $\left\langle d, e, f, z_{1}, \ldots, z_{k}\right\rangle$. If $\operatorname{deg}_{z_{k}} q=0$, then $a q \sim_{k} q a$ and thus $\overleftarrow{w}_{z} \sim_{k}$ $\overleftarrow{v}_{z}$. If, however, $\operatorname{deg}_{z_{k}} q>0$ then let $q=t_{1} z_{k}^{i_{1}} t_{2} z_{k}^{i_{2}} \cdots z_{k}^{i_{l}} t_{l+1}$, for some positive integers $i_{j}, l$, and $t_{i} \in\left\langle d, e, f, z_{1}, \ldots, z_{k-1}\right\rangle$. We only need to show that aqa $\sim_{k} q a$. This is clear, since for $i>0$ and for any $x \in\left\langle d, e, f, z_{1}, \ldots, z_{k-1}\right\rangle$ we have $z_{k}^{i} x a \sim_{k} z_{k} a x \sim_{k} a z_{k} a x \sim_{k}$ $a z_{k} x a \sim_{k} a z_{k}^{i} x a$. Thus $\overleftarrow{w}_{z} \sim_{k} \overleftarrow{v}_{z}$

- Finally, if $w=r z a z t, v=$ razat we proceed analogously as in the previous subcase.

We have proved (4.5). Thus the assignment $w \mapsto \overleftarrow{w}_{z}$ determines a function $\phi_{k+1}$ from the subset $\left[W_{k+1}\right.$ ] of all classes of words in $W_{k+1}$ with respect to $\mathrm{HK}_{\Theta_{k+1}}$ to the monoid $\mathrm{HK}_{\Theta_{k}}$. Clearly, the class of any element of $\left\langle a c b, f e d z_{1} \cdots z_{k} z_{k+1}\right\rangle$ in $\mathrm{HK}_{\Theta_{k+1}}$ is mapped to a unique class of an element of the submonoid $\left\langle a c b, f e d z_{1} \cdots z_{k}\right\rangle$, which is free in $\mathrm{HK}_{\Theta_{k}}$ by the induction hypothesis. Thus, also the submonoid $\left\langle[a c b],\left[\operatorname{fed} z_{1} \cdots z_{k} z_{k+1}\right]\right\rangle$ of $\mathrm{HK}_{\Theta_{k+1}}$ must be free.

We are now ready to prove the general statement of this example. Assume that the assertion is satisfied for some $\Theta_{n, k, m}$, with $n, m \geq 3, k \geq 1$. Let $F=\left\langle V\left(\Theta_{n, k, m}\right)\right\rangle$ and $F^{\prime}=\left\langle V\left(\Theta_{n+1, k, m}\right)\right\rangle$ be free monoids. Consider the set $W$ of all words in $F^{\prime}$ that are both $\Phi_{n+1}$-free and $\Psi_{m}$-free. As in the case of graphs $\Theta_{k}$ one can easily argue that $W$ is closed with respect to equivalence classes of $\sim_{n+1, k, m}$. The remaining argument is similar to the one used in the special case $n=m=3$. This time we only need to assign to every word $w \in W$ a maximal subword $v$ such that $\operatorname{deg}_{a_{n+1}} w=0$. This yields a function from the subset of the monoid $\mathrm{HK}_{\Theta_{n+1, k, m}}$ to the monoid $\mathrm{HK}_{\Theta_{n, k, m}}$ such that the classes of elements of the monoid $\left\langle a_{1} a_{n+1} a_{n} \cdots a_{2}, b_{m} b_{m-1} \cdots b_{1} z_{1} \cdots z_{k}\right\rangle$ are mapped to unique classes of the monoid $\left\langle a_{1} a_{n} \cdots a_{2}, b_{m} b_{m-1} \cdots b_{1} z_{1} \cdots z_{k}\right\rangle$, which is free by the induction hypothesis. The assertion for $\Theta_{n+1, k, m}$ follows. 
By symmetric arguments we can verify the freeness property of the appropriate submonoid of $\mathrm{HK}_{\Theta_{n, k, m+1}}$. Since we already know that the assertion follows for all graphs $\Theta_{3, k+1,3}$, with $k \geq 0$, the general assertion follows.

Here is a remark. One might try to use an approach based on the diamond lemma in order to find the normal form of all elements of $\mathrm{HK}_{\Theta}$. However, this typically leads to infinite Gröbner bases. For instance, in Example 4 it is possible to introduce the following system $S$ of reductions in $\mathrm{K}\langle a, b, c, d, e, f\rangle$ with respect to the natural deg-lex order (see [5] for terminology and the necessary background):
(1) $(a a, a)$
(2) $(b b, b)$,
(3) $(c c, c)$,
(4) $(d d, d)$,
(5) $(e e, e)$,
(6) $(f f, f)$,
(7) $(a b a, a b)$,
(8) $(b a b, a b)$,
(9) $(a c a, c a)$,
(10) (cac, ca),
(11) $(b c b, b c)$,
(12) $(c b c, b c)$,
(13) $(d a d, d a)$,
(14) $(a d a, d a)$,
(15) (ded,de)
(16) (ede, de),
(17) $(d f d, f d)$,
(18) $(f d f, f d)$,
(19) (efe,ef),
(20) (fef,ef),
(21) (ea,ae),
(22) (fa,af),
(23) $(d b, b d)$,
(24) $(e b, b e)$,
(25) $(f b, b f)$,
(26) $(d c, c d)$,
(27) $(e c, c e)$,
(28) $(f c, c f)$,
(29) (bwdab,wdab), (30) (cwdac, cwda),

$$
\text { for } w \in\{1, f, e f\} \cdot\langle d e f\rangle \cup\{1, e, f e\} \cdot\langle d f e\rangle,
$$

(31) (edawe, dawe),

(32) (fdawf, fdaw),

$$
\text { for } w \in\langle c b a\rangle \cdot\{1, c, c b\} \cup\langle b c a\rangle \cdot\{1, b, b c\} \text {, }
$$

(33) (awda, wda),

$$
\text { for } w \in\{1, c\} \cdot\{1, f, e f\} \cdot\langle d e f\rangle \cup\{1, c\} \cdot\{1, e, f e\} \cdot\langle d f e\rangle \text {, }
$$

(34) (dawd, daw),

$$
\text { for } w \in\langle b c a\rangle \cdot\{1, b, b c\} \cdot\{1, e\} \cup\langle c b a\rangle \cdot\{1, c, c b\} \cdot\{1, e\} \text {. }
$$

Then one can show, though through a quite involved and detailed caseby-case investigation, that all ambiguities that arise from the system $S$ (more than 150) are in fact resolvable. This of course implies that different elements of the monoid $\langle a c b, f e d\rangle$ belong to different equivalence classes of $\sim$. This approach, however, seems inapplicable to the general case of graphs $\Theta_{n, k, m}$, considered in Example 5.

\section{The proof of Theorem 1}

With all previous examples studied, we are ready to prove the main result of this paper.

Proof of Theorem 1: Let $F=\langle V(\Theta)\rangle$. The implication $(1) \Rightarrow(2)$ is proved by induction on $r:=|V(\Theta)|$. If $|V(\Theta)| \leq 2$ then $\Theta$ is acyclic 
and obviously the monoid $\mathrm{HK}_{\Theta}$ is finite, hence $A_{\Theta}$ is a PI-algebra. We proceed to the inductive step. If $\Theta_{1}$ is a connected component of $\Theta$ and $\Theta_{2}=\Theta \backslash \Theta_{1} \neq \emptyset$, then $\mathrm{HK}_{\Theta}$ is a direct product of $\mathrm{HK}_{\Theta_{1}}$ and $\mathrm{HK}_{\Theta_{2}}$, so that $A_{\Theta}=A_{\Theta_{1}} \otimes A_{\Theta_{2}}$. Therefore, $A_{\Theta}$ is a PI-algebra by Theorem 6.1.1 in [17], because so are the algebras $A_{\Theta_{i}}, i=1,2$, by the induction hypothesis. Thus, we may assume that $\Theta$ is a connected graph.

Suppose that $\Theta$ has a source vertex. Denote it by $a$. We know that $a w a=a w$, for each $w \in \mathrm{HK}_{\Theta}$, see Remark 5. Let $\Theta^{\prime}$ arise from $\Theta$ by removing the vertex $a$ and all arrows that originate from $a$. We can assume that $A_{\Theta^{\prime}} \subseteq A_{\Theta}$ and therefore:

$$
A_{\Theta}=A_{\Theta^{\prime}}+A_{\Theta^{\prime}} a A_{\Theta^{\prime}}=A_{\Theta^{\prime}}+\underbrace{a\left(A_{\Theta^{\prime}} a A_{\Theta^{\prime}}\right)}_{a A_{\Theta^{\prime}}}+(1-a) A_{\Theta^{\prime}} a A_{\Theta^{\prime}} .
$$

The summand $I:=(1-a) A_{\Theta^{\prime}} a A_{\Theta^{\prime}}$ is clearly an ideal in $A_{\Theta}$ and $I^{2}=0$. Let $\bar{x}$ be the image of $x \in A_{\Theta}$ under the natural map $A_{\Theta} \longrightarrow A_{\Theta} / I$. Then $\overline{A_{\Theta}}=\overline{A_{\Theta^{\prime}}}+\overline{a A_{\Theta^{\prime}}}$, which is a finitely generated right $\overline{A_{\Theta^{\prime}}}$-module. By the inductive hypothesis we may assume that $\overline{A_{\Theta^{\prime}}}$ (in fact isomorphic to $\left.A_{\Theta^{\prime}}\right)$ is a PI-algebra, so from [14, Corollary 13.4.9], it follows that $\overline{A_{\Theta}}$ is PI as well. This, of course, implies that $A_{\Theta}$ is PI. The same argument works in the case when $a$ is a sink vertex.

Therefore, we may assume that $\Theta$ neither contains sink nor source vertices. Then, by condition (1), it must be a cycle. Thus, by Example 2 we have that $A_{\Theta}$ is a PI-algebra, see [18], which yields the first implication.

The implication $(2) \Rightarrow(3)$ is well known, see [12, Corollary 10.7]. The implication $(3) \Rightarrow(4)$ is clear. So, we are left with proving $(4) \Rightarrow$ (1). Assume, contradictory to (1), that the graph $\Theta$ contains two cycles that are connected by an oriented path. Namely, we have two different sets of vertices $\mathcal{A}=\left\{a_{1}, \ldots, a_{n}\right\}$ and $\mathcal{B}=\left\{b_{1}, \ldots, b_{m}\right\}$ of $\Theta$ such that the induced subgraphs $\Theta_{1}, \Theta_{2}$ of $\Theta$ with the respective vertex sets $\mathcal{A}$, $\mathcal{B}$ are cycles of the form $a_{1} \rightarrow a_{2} \rightarrow \cdots \rightarrow a_{n} \rightarrow a_{1}$ and $b_{1} \rightarrow b_{2} \rightarrow$ $\cdots \rightarrow b_{m} \rightarrow b_{1}$. If $\mathcal{A} \cap \mathcal{B} \neq \emptyset$, then assume that this intersection contains an element $c$. We can assume that $c=a_{1}=b_{1}$ (perhaps we need to renumber $\left.a_{i}, b_{j}\right)$. By the same arguments as in Example 3 we argue that all elements of the submonoid of $F$ generated by $a=a_{n} a_{n-1} \cdots a_{1}$ and $b=b_{m} b_{m-1} \cdots b_{1}$ are essentially non-rewritable in $\mathrm{HK}_{\Theta}$, except for the edge relations of the form $x=x x$. Thus $\langle[a],[b]\rangle$ is a free submonoid in $\mathrm{HK}_{\Theta}$, which contradicts (3). If we are in the case when $\mathcal{A} \cap \mathcal{B}=\emptyset$, we know that $\Theta$ contains a subgraph of the form $\Theta_{n, k, m}$ introduced in Example 5, and thus it is clear that the free submonoid of $\mathrm{HK}_{\Theta_{n, k, m}}$ is a submonoid of $\mathrm{HK}_{\Theta}$. This again contradicts (4) and the proof is complete. 
We conjecture that $\operatorname{GKdim}\left(A_{\Theta}\right)$ always is an integer if it is finite. More generally, one might ask whether $A_{\Theta}$ is an automaton algebra in the sense of Ufnarovskii [22] for any Hecke-Kiselman monoid $\mathrm{HK}_{\Theta}$ and whether Theorem 1 can be generalized to the entire class of such monoids.

Acknowledgement. The work of the second author is supported by National Science Centre grant 2016/23/B/ST1/01045 (Poland).

\section{References}

[1] D. J. AnICK, On monomial algebras of finite global dimension, Trans. Amer. Math. Soc. 291(1) (1985), 291-310. DOI : 10.2307/1999910.

[2] R. Aragona and A. D'Andrea, Hecke-Kiselman monoids of small cardinality, Semigroup Forum 86(1) (2013), 32-40. DOI : 10.1007/s00233-012-9422-2.

[3] D. N. Ashikhmin, M. V. Volkov, and W. T. Zhang, The finite basis problem for Kiselman monoids, Demonstr. Math. 48(4) (2015), 475-492. DOI: 10.1515/dema-2015-0035.

[4] J. P. Bell and J. Z. GonÇAlves, Free algebras and free groups in Ore extensions and free group algebras in division rings, J. Algebra 455 (2016), 235-250. DOI: 10.1016/j.jalgebra.2016.02.011.

[5] G. M. Bergman, The diamond lemma for ring theory, Adv. in Math. 29(2) (1978), 178-218. DOI : 10.1016/0001-8708(78)90010-5.

[6] B. BRINK AND R. B. Howlett, A finiteness property and an automatic structure for Coxeter groups, Math. Ann. 296(1) (1993), 179-190. DOI: 10.1007/BF01445101.

[7] E. Collina And A. D'Andrea, A graph-dynamical interpretation of Kiselman's semigroups, J. Algebraic Combin. 41(4) (2015), 1115-1132. DOI: 10.1007/ s10801-014-0569-7.

[8] T. Denton, F. Hivert, A. Schilling, and N. M. Thiéry, On the representation theory of finite $\mathcal{J}$-trivial monoids, Sém. Lothar. Combin. 64 (2010/11), Art. B64d, 44 pp.

[9] L. Forsberg, Effective representations of Hecke-Kiselman monoids of type A, Preprint (2017). arXiv:1205.0676.

[10] O. Ganyushkin and V. Mazorchuk, On Kiselman quotients of 0-Hecke monoids, Int. Electron. J. Algebra 10 (2011), 174-191.

[11] P. DE la HARPe, Groupes de Coxeter infinis non affines, Exposition. Math. 5(1) (1987), 91-96.

[12] G. R. Krause and T. H. Lenagan, "Growth of Algebras and Gelfand-Kirillov Dimension", Research Notes in Mathematics 116, Pitman (Advanced Publishing Program), Boston, MA, 1985.

[13] G. Kudryavtseva and V. Mazorchuk, On Kiselman's semigroup, Yokohama Math. J. 55(1) (2009), 21-46.

[14] J. C. McConnell and J. C. Robson, "Noncommutative Noetherian Rings", With the cooperation of L. W. Small, Pure and Applied Mathematics (New York), A Wiley-Interscience Publication, John Wiley \& Sons, Ltd., Chichester, 1987.

[15] J. OkniŃski, "Semigroup Algebras", Monographs and Textbooks in Pure and Applied Mathematics 138, Marcel Dekker, Inc., New York, 1991. 
[16] J. OKniŃski And A. SALwa, Generalised Tits alternative for linear semigroups, J. Pure Appl. Algebra 103(2) (1995), 211-220. DOI: 10.1016/0022-4049(95) 00104-5.

[17] L. H. Rowen, "Polynomial Identities in Ring Theory", Pure and Applied Mathematics 84, Academic Press, Inc. [Harcourt Brace Jovanovich, Publishers], New York-London, 1980.

[18] L. W. Small, J. T. Stafford, and R. B. Warfield, Jr., Affine algebras of Gelfand-Kirillov dimension one are PI, Math. Proc. Cambridge Philos. Soc. 97(3) (1985), 407-414. DOI : 10.1017/S0305004100062976.

[19] J. Tits, Le problème des mots dans les groupes de Coxeter, in: "Symposia Mathematica" (INDAM, Rome, 1967/68), Vol. 1, Academic Press, London, 1969, pp. $175-185$.

[20] S. V. Tsaranov, Representation and classification of Coxeter monoids, European J. Combin. 11(2) (1990), 189-204. DOI : 10.1016/S0195-6698(13)80073-X.

[21] V. A. UfnarovskiI, A growth criterion for graphs and algebras defined by words, (Russian), Mat. Zametki 31(3) (1982), 465-472, 476; English translation: Math. Notes 31(3) (1982), 238-241.

[22] V. A. Ufnarovski, Combinatorial and asymptotic methods in algebra, (Russian), in: "Algebra - 6", Itogi Nauki i Tekhniki. Ser. Sovrem. Probl. Mat. Fund. Napr. 57, Vsesoyuz. Inst. Nauchn. i Tekhn. Inform., Moscow, 1990, pp. 5-177.

Institute of Mathematics, University of Warsaw, Banacha 2, 02-097 Warsaw, Poland

E-mail address: a.mecel@mimuw.edu.pl

E-mail address: okninski@mimuw.edu.pl

Primera versió rebuda el 5 de maig de 2017, darrera versió rebuda el 8 de gener de 2018. 